\title{
Quantitative pathological changes in the cerebellum of multiple system atrophy
}

\author{
Richard A. Armstrong \\ Vision Sciences, Aston University, Birmingham, UK
}

\begin{abstract}
Multiple system atrophy (MSA) is a rare neurodegenerative disorder associated with parkinsonism, ataxia, and autonomic dysfunction. Its pathology is primarily subcortical comprising vacuolation, neuronal loss, gliosis, and $\alpha$-synucleinimmunoreactive glial cytoplasmic inclusions (GCI). To quantify cerebellar pathology in MSA, the density and spatial pattern of the pathological changes were studied in $\alpha$-synuclein-immunolabelled sections of the cerebellar hemisphere in 10 MSA and 10 control cases. In MSA, densities of Purkinje cells $(P C)$ were decreased and vacuoles in the granule cell layer (GL) increased compared with controls. In six MSA cases, GCI were present in cerebellar white matter. In the molecular layer (ML) and GL of MSA, vacuoles were clustered, the clusters exhibiting a regular distribution parallel to the edge of the folia. Purkinje cells were randomly or regularly distributed with large gaps between surviving cells. Densities of glial cells and surviving neurons in the ML and surviving cells and vacuoles in the GL were negatively correlated consistent with gliosis and vacuolation in response to neuronal loss. Principal components analysis (PCA) suggested vacuole densities in the ML and vacuole density and cell losses in the GL were the main source of neuropathological variation among cases. The data suggest that: (1) cell losses and vacuolation of the GCL and loss of PC were the most significant pathological changes in the cases studied, (2) pathological changes were topographically distributed, and (3) cerebellar pathology could influence cerebral function in MSA via the cerebello-dentato-thalamic tract.
\end{abstract}

Key words: multiple system atrophy, cerebellum, vacuolation, $\alpha$-synuclein, spatial pattern.

\section{Introduction}

Multiple system atrophy (MSA) is a rare, largely sporadic neurodegenerative disorder, associated with varying degrees of parkinsonism, ataxia, and autonomic dysfunction [25]. The average annual incidence of the disorder is 3.0/100,000 of the population and median survival time is 8.5 years [12]. Symptoms of MSA begin early in the fifth decade and the disorder is slightly more common in males than in females (male : female $1.3: 1$ ) [37]. Two main subtypes of the disease are recognised: the cerebellar subtype (MSA-C) and the parkinsonian subtype (MSA-C) [1921]. A third subtype, viz. Shy-Drager syndrome, in which the symptoms are primarily autonomic, is not currently included as a subtype of MSA [21].

The neuropathology of MSA largely affects subcortical grey matter including the substantia nigra, striatum, inferior olivary nucleus, pontine nuclei, and cerebellum $[8,9,17,25]$. In some cases, there is a progressive cerebral atrophy affecting the frontal lobes [22] and the motor/premotor areas [36]. Histological- 
ly, MSA is characterised by selective neuronal loss, gliosis, and myelin pathology [25], the 'signature' pathological lesion being the glial cytoplasmic inclusion (GCl) found mainly in oligodendrocytes [28]. The $\mathrm{GCl}$ are composed of argyrophilic 10-15 nm diameter coated filaments immunoreactive for ubiquitin and $\alpha$-synuclein, but glial fibrillar acid protein (GFAP) reactivity is absent [34]. $\alpha$-Synuclein-immunoreactive neuronal cytoplasmic inclusions $(\mathrm{NCl})$ have also been observed in MSA but at significantly lower densities $[8,16,17,29]$. $\alpha$-Synuclein is a small pre-synaptic protein that regulates the normal functioning of dopamine transporter and tyrosine hydroxylase [24]. It normally exists in a relatively unfolded state and is highly soluble, but in synucleinopathies such as MSA it, undergoes a conformational change to insoluble amyloid fibrils that form a major component of the $\mathrm{GCl}$.

Cerebellar pathology has been reported in previous studies of MSA $[18,20,27]$ including loss of Purkinje cells (PC) [26] and the presence of $\alpha$-synucleinimmunoreactive cellular inclusions in the molecular layer (ML) [31,33]. Cerebellar pathology could influence a variety of brain functions in MSA including motor function, the fine timing of events, sensory analysis, feeding behaviour, the modulation of cognition, and the regulation of emotion [22]. Hence, to quantify cerebellar pathology in MSA and identify the anatomical pathways likely to be affected, the density and spatial pattern of vacuoles, surviving neurons, glial cell nuclei, and glial cytoplasmic inclusions (GCl) were studied in $\alpha$-synuclein-immu- nolabelled sections of the cerebellar hemisphere in 10 MSA and 10 control cases. The specific objectives were: (1) to quantify and compare pathological changes in the cerebellar hemisphere in MSA and cognitively normal brain, (2) to determine the spatial topography of the pathological changes within each layer, (3) to examine the spatial correlations between the vacuoles, glial cell nuclei, and $\mathrm{GCl}$ both within and between layers, (4) to investigate pathological differences among cases, and (5) to consider how cerebellar pathology might affect cerebral function in MSA.

\section{Material and methods \\ Cases}

Ten cases of MSA (details in Table I) and 10 control cases (50-80 years of age) were obtained from the Brain Bank, Department of Neuropathology, Institute of Psychiatry, King's College London, UK. Control cases had no neurological or psychiatric histories and were matched as closely as possible for gender and age to the MSA cases. Multiple system atrophy cases were diagnosed according to the Minneapolis Consensus Criteria [19-21] and subsequently neuropathologically verified. All cases had $\mathrm{GCl}$ in subcortical grey matter, including the striatum, substantia nigra, pontine nuclei, and medulla [8]. The major clinical features of the 10 cases are shown in Table II. Four cases were diagnosed as the MSA-C subtype and two as the MSA-P subtype. Four cases had a more complex pathology, exhibiting both

Table I. Subtypes, demographic data, and brain weights (BW) of the multiple system atrophy (MSA) cases studied

\begin{tabular}{|c|c|c|c|c|c|c|}
\hline Case & Subtype & Sex & Onset (years) & Death (years) & Duration (years) & BW (gm) \\
\hline A & MSA-P & $\mathrm{F}$ & 73 & 77 & 4 & 1274 \\
\hline B & $M$ & $\mathrm{Ma}$ & 69 & 76 & 7 & 1290 \\
\hline C & MSA-C & Ma & 63 & 65 & 2 & 1276 \\
\hline D & MSA-C & $\mathrm{Ma}$ & 65 & 67 & 2 & 1309 \\
\hline$E$ & MSA-C & $\mathrm{F}$ & 70 & 81 & 11 & 817 \\
\hline $\mathrm{F}$ & MSA-P & Ma & 66 & 78 & 12 & 1400 \\
\hline G & MSA-C & $\mathrm{Ma}$ & 69 & 72 & 3 & 1035 \\
\hline $\mathrm{H}$ & $M$ & $\mathrm{~F}$ & 61 & 63 & 2 & 1283 \\
\hline I & $M$ & Ma & 63 & 73 & 10 & NA \\
\hline J & $M$ & Ma & NA & 53 & NA & NA \\
\hline
\end{tabular}

MSA-P - Parkinsonian subtype, MSA-C - cerebellar subtype, $M$ - mixed pathology, i.e. cases with features of more than one subtype, Ma - male, $F$ - female, NA - data not available 
Table II. Clinical features of the multiple system atrophy cases studied

\begin{tabular}{|ll|}
\hline Case & Clinical features \\
\hline A & $\begin{array}{l}\text { Rigidity and akinesia. No cerebellar ataxia. Nocturnal sweating, salivation, and frequent urination. Cognition preserved. } \\
\text { Perseveration of speech }\end{array}$ \\
\hline B & $\begin{array}{l}\text { Minimal parkinsonism. Balance problems. Impotence, urination and swallowing difficulties, impaired blinking. Cognition } \\
\text { preserved. Depression }\end{array}$ \\
\hline C & $\begin{array}{l}\text { Minimal parkinsonism. Restricted mobility and falls. Cerebellar ataxia. Urination difficulty. Cognition preserved. } \\
\text { Dysarthria }\end{array}$ \\
\hline D & Some tremor of hands. Significant cerebellar ataxia. Dysarthria \\
\hline E & Minimal parkinsonism. Significant cerebellar ataxia affecting limbs and trunk. Memory impairment \\
\hline F & Significant parkinsonism with tremor and cogwheel rigidity. Urination and swallowing difficulties. Cognition preserved \\
\hline G & Minimal parkinsonism. Significant cerebellar ataxia. Confusion \\
\hline H & Significant parkinsonism and cerebellar ataxia. Cognition preserved \\
\hline I & Some rigidity. Significant cerebellar ataxia. Swallowing difficulties. Cognition preserved. Dysarthria \\
\hline J & Significant parkinsonism and cerebellar ataxia. Dysphagia. Cognition preserved. \\
\hline
\end{tabular}

parkinsonism and cerebellar clinical signs, and could not easily be assigned to either the MSA-C or MSA-P subtypes. Hence, the two MSA-P cases exhibited significant parkinsonism but no cerebellar ataxia, the four MSA-C cases showed significant cerebellar ataxia but with minimum parkinsonism, while the four 'mixed' cases exhibited a combination of cerebellar and parkinsonian symptoms. Only cases of the MSA-C subtype exhibited evidence of cognitive impairment including memory impairment and confusion.

\section{Histological methods}

After death, consent of the next of kin was obtained for brain removal following local Ethical Committee procedure and the 1964 Declaration of Helsinki (as revised in Edinburgh, 2000). A block of the right cerebellar cortex was taken from each case at the level of the superior cerebellar peduncle. Tissue was fixed in 10\% phosphate-buffered formal-saline and embedded in paraffin wax. For quantitative analysis, sequential coronal 7- $\mu \mathrm{m}$ sections were stained with haematoxylin and eosin (H/E) or immunohistochemistry (IHC) was performed using a non-phosphorylated polyclonal rabbit antibody (a116), after formic acid pretreatment, and at a dilution 1/3000, against the 116-131 amino acid sequence of $\alpha$-synuclein (kindly supplied by $\operatorname{Dr} \mathrm{D}$. Hanger). This type of antibody is regarded as one of the most efficient available, especially for revealing the $\mathrm{GCl}$, and is particularly recommended for diagnostic use [15].
The secondary antibody was biotinylated anti-rabbit antibody (DAKO diagnostics, Germany), used at a concentration of $1 / 200$, which binds to the avidinperoxidase complex. Chromogen 3,3-diaminobenzidene tetrahydrochloride was used to reveal the $\mathrm{GCl}$. Immunolabelled sections were also stained with haematoxylin.

\section{Morphometric methods}

Variations in density of histological features were measured parallel to the edge of randomly selected

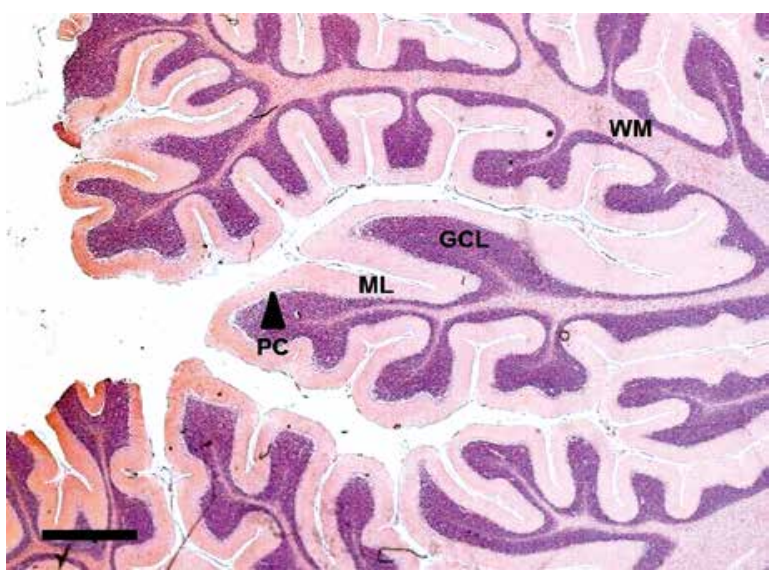

Fig. 1. Section of cerebellar hemisphere in a case of multiple system atrophy (MSA) ( $\alpha$-synuclein immunohistochemistry, haematoxylin; Magnification bar = $1 \mathrm{~mm})$. GCL - granule cell layer, ML - molecular layer, PC - Purkinje cell layer, WM - white matter. 


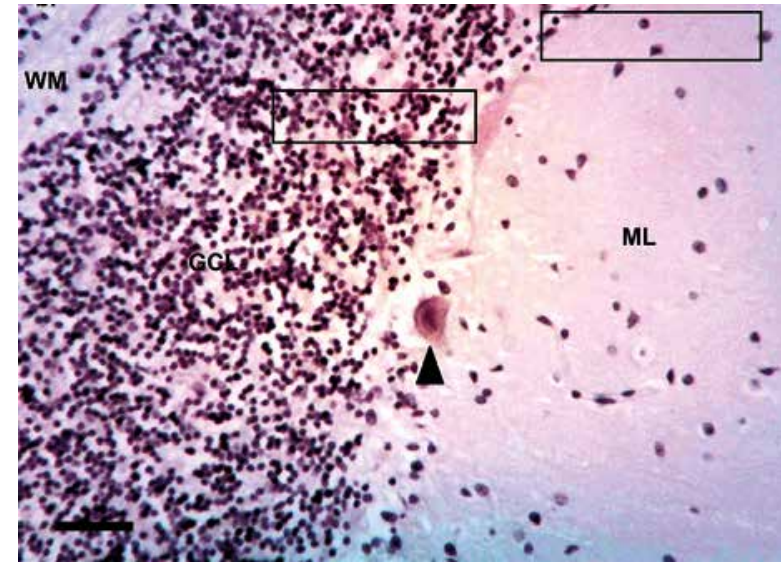

Fig. 2. Quantitative method of sampling the cerebellum hemisphere in multiple system atrophy (MSA) showing the $50 \times 250 \mathrm{~mm}$ plots. $(\alpha-$ synuclein immunohistochemistry, haematoxylin; Magnification bar $=50 \mathrm{~mm}$ ). Arrow indicates surviving Purkinje cell. GCL - granule cell layer, $\mathrm{ML}$ - molecular layer, PC - Purkinje cell layer.

folia within each case (Fig. 1). Within each folium, a strip of cerebellar cortex 3200 to $4800 \mu \mathrm{m}$ in length, starting at a randomly determined location, was studied with $64-96,50 \times 250 \mu \mathrm{m}$ sample fields arranged contiguously (Fig. 2) [3]. First, the sample field was positioned with the shorter dimension aligned along the upper edge of the GL at the base of the PC layer to quantify the density of PC and the pathology of the inner region of the ML. In each sam-

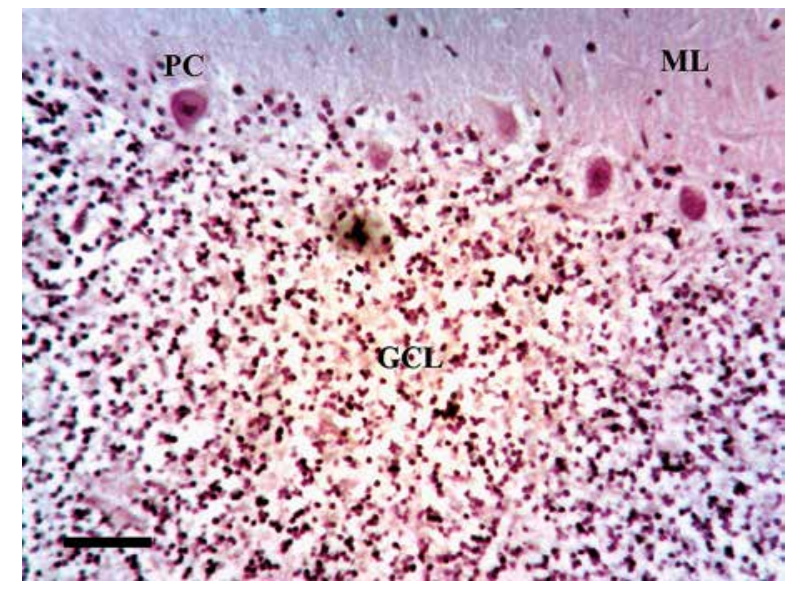

Fig. 3. Vacuolation in a case of multiple system atrophy (MSA) ( $\alpha$-synuclein immunohistochemistry, haematoxylin; magnification bar $=20 \mathrm{~mm}$ ). $\mathrm{GCL}$ - granule cell layer, ML - molecular layer, $P C$ - Purkinje cell layer. ple field, the number of PC, distinct vacuoles greater than $5 \mu \mathrm{m}$ in diameter, neurons, glial cell nuclei, and $\alpha$-synuclein-immunoreactive inclusions were counted. Second, at the same position, the field was moved to sample the outer region of the $G L$, the short edge of the field aligned with the edge of the granule cells (Fig. 2). It was not possible in these preparations to differentiate between different cell types in the GL, e.g. granule cells, Golgi type II cells, glia, and a single count of cell density was made. Third, at the same location, the number of vacuoles, glial cell nuclei, and $\mathrm{GCl}$ (Fig. 3 and 4) were counted in sample fields arranged along the white matter, the upper short edge of the sample field being aligned with the lower edge of the GL (Fig. 2).

\section{Data analysis}

Data analysis was carried out using STATISTICA software (Statsoft Inc., 2300 East 14th St, Tulsa, Ok, 74104, USA). First, densities of histological features in the $M L, G L$, and white matter were compared in MSA and control subjects using a 't' test. Second, the spatial pattern of a histological feature, i.e. whether the feature was distributed randomly, regularly, or in clusters, was determined using the variance/mean (V/M) method described previously $[1,2,5,6]$. Third, spatial correlations between histological features along the folia were tested in each case using Pearson's correlation coefficient (' $r$ ') [4]. Fourth, to study pathological variation among cases, the data were

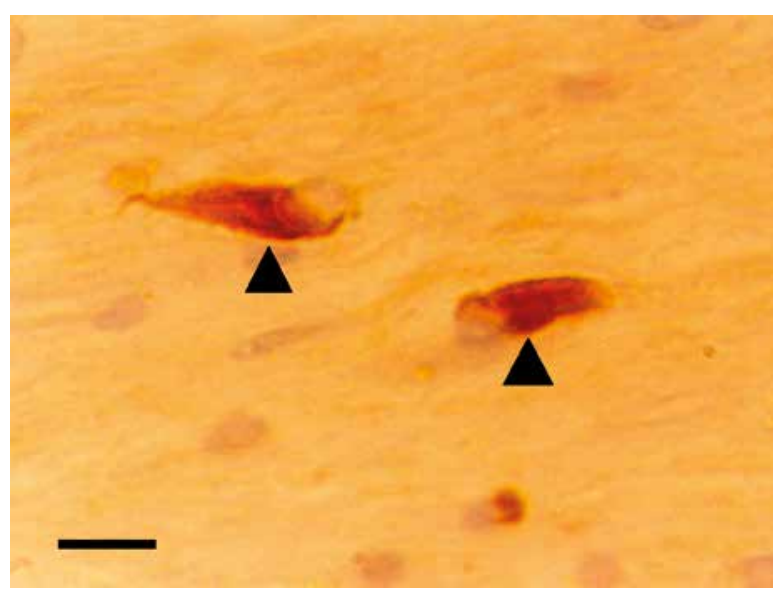

Fig. 4. High-power view of glial cytoplasmic inclusions (GCl) (arrows) in the white matter of the cerebellum of a case of multiple system atrophy (MSA) ( $\alpha$-synuclein immunohistochemistry, haematoxylin; magnification bar $=6 \mathrm{~mm}$ ). 
analysed using principal components analysis (PCA) [11]. The result of a PCA is a plot of the ten MSA cases in relation to the extracted $P C$ in which distance between cases reflects their pathological similarity or dissimilarity. To correlate the location of a case on a PC axis with the numerical density of a specific histological feature, correlations (Pearson's ' $r$ ') were calculated between the densities of each histological feature and the factor loadings of cases on PC1 and PC2. Clinical features were also plotted onto the PCA to determine if cases were segregated according to clinical symptoms in relation to PC1 and PC2.

\section{Results}

Pathological features observed in MSA included: (1) modest vacuolation of the ML in some cases and more extensive vacuolation of the $G L$, (2) loss of PC, and (3) $\mathrm{GCl}$ in white matter (Fig. 1-4). No $\alpha$-synuclein-immunoreactive $\mathrm{GCl}$ or $\mathrm{NCl}$ were observed in the $M L$ or GL. Mean density of vacuoles in the GL was significantly increased $(t=2.57, p<0.05)$ and $P C$ was decreased ( $t=7.65, p<0.001)$, in MSA compared with controls (Fig. 5). In addition, the mean density of vacuoles was significantly greater in the GCL compared with the $M L(t=3.52, p<0.01)$ but was similar to vacuole density in adjacent white matter $(t=2.01$, $p>0.05)$.

Examples of the spatial patterns of histological features along the folia are shown in Fig. 6. The V/M of the PC was not significantly different to unity at any field size, suggesting a random distribution. The $V / M$ of the vacuolation in the $M L$, however, revealed significant peaks at field sizes $100 \mathrm{~mm}$ and $400 \mathrm{~mm}$, suggesting clustering at two scales in the tissue, i.e. vacuoles were clustered, the mean dimension of the clusters being equal to $100 \mathrm{~mm}$, and they were regularly distributed along the folium, the smaller clusters being aggregated into larger clusters, $400 \mathrm{~mm}$ in diameter.

The spatial patterns of all histological features in each case are shown in Table III. Vacuoles were clustered in the ML in the majority of cases, regular spaced clustering of vacuoles along the folia being present in 4/10 (40\%) cases. Similarly, neurons in the $\mathrm{ML}$ were clustered, a regular distribution of clusters being present in $6 / 10$ (60\%) cases. By contrast, glial cell nuclei in the $M L$ were randomly or regularly distributed. In the majority of cases, PCs were randomly or regularly distributed and there were large gaps between surviving cells. In the GL, the vacuoles and cell nuclei were clustered, a regular distribution of clusters being present. In the white matter, large clusters of vacuoles were present and the $\mathrm{GCl}$ and glial cell nuclei exhibited a regular distribution of clusters along the folia. The frequency of the different types of spatial pattern was similar in the different layers $\left(\chi^{2}=5.28,6 \mathrm{DF}, p>0.05\right)$.

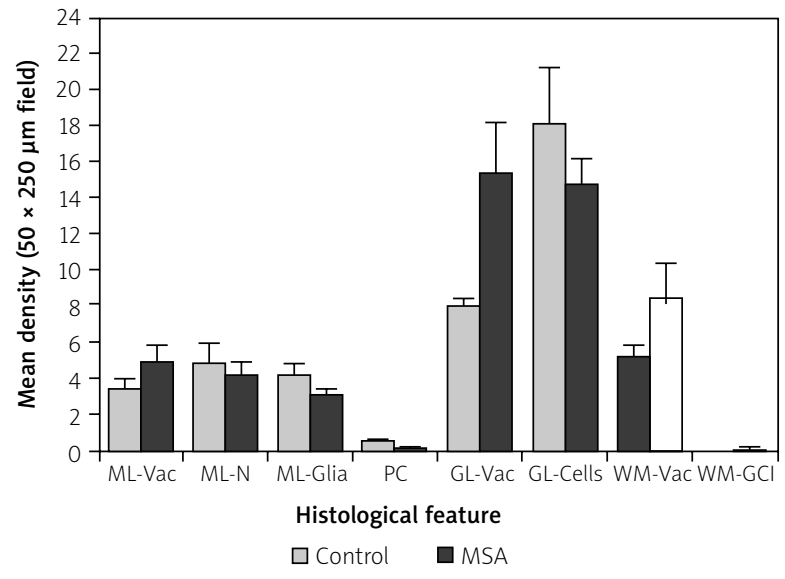

Fig. 5. Mean densities $(50 \times 250 \mathrm{~mm}$ field, standard error of mean in parentheses) of histological features (Vac - vacuolation, PC - Purkinje cells, $\mathrm{GCl}$ - glial cytoplasmic inclusions) in various layers of the cerebellar cortex ( $\mathrm{ML}$ - molecular layer, PC - Purkinje cell layer, GL - granule cell layer, WM - white matter) in ten cases of multiple system atrophy (MSA) and ten control cases.

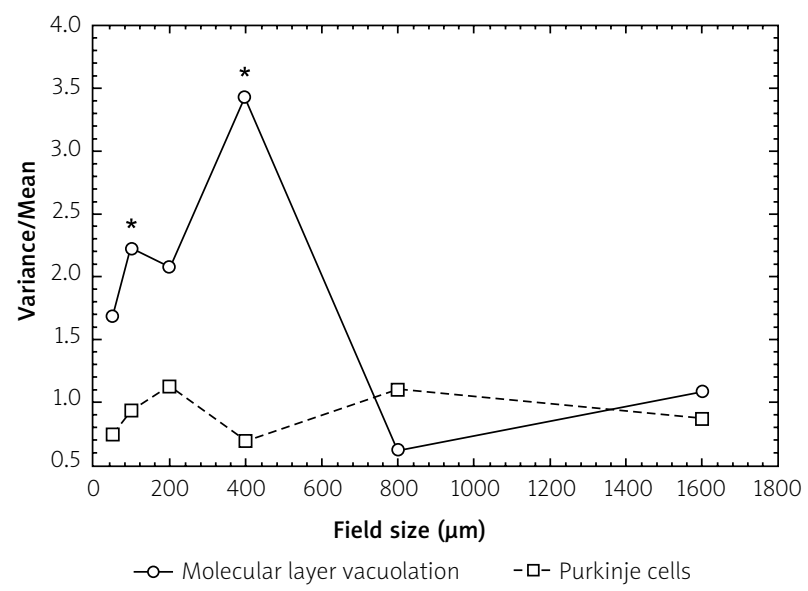

Fig. 6. Examples of the topographical patterns of the vacuolation and glial cell nuclei in the cerebellum in a case of multiple system atrophy (MSA) (Case A). *Significant variance/mean peaks. 
Table III. Spatial patterns of histological features in various layers of the cerebellar cortex in 10 cases of multiple system atrophy

\begin{tabular}{|c|c|c|c|c|c|c|c|c|c|}
\hline \multirow[t]{2}{*}{ Case } & \multicolumn{3}{|c|}{ Molecular layer (ML) } & \multicolumn{3}{|c|}{ Granule cell layer (GL) } & \multicolumn{3}{|c|}{ White matter (WM) } \\
\hline & Vac & SN & G & PC & Vac & $\mathrm{SC}$ & Vac & $\mathrm{GCl}$ & G \\
\hline$A$ & $\begin{array}{l}100 \\
400\end{array}$ & $\mathrm{R}$ & Reg & $\mathrm{R}$ & 200 & 200 & $R$ & - & Reg \\
\hline B & $\begin{array}{c}50 \\
400\end{array}$ & $\mathrm{R}$ & Reg & $\mathrm{R}$ & $>800$ & $\mathrm{R}$ & 400 & - & 200 \\
\hline C & $R$ & 200 & $\mathrm{R}$ & Reg & $>800$ & 400 & $>800$ & Reg & 400 \\
\hline $\mathrm{D}$ & $>800$ & $>400$ & $>400$ & Reg & $>400$ & $>800$ & $>400$ & 100 & $>40$ \\
\hline$E$ & 200 & $\begin{array}{c}50 \\
400\end{array}$ & $R$ & Reg & 100 & $\mathrm{R}$ & $>800$ & - & $\mathrm{R}$ \\
\hline $\mathrm{F}$ & $>800$ & 400 & $>800$ & Reg & 400 & Reg & 200 & - & 40 \\
\hline$G$ & $>400$ & $>400$ & $R$ & - & $>400$ & $>400$ & $>400$ & $R$ & $>400$ \\
\hline $\mathrm{H}$ & $\begin{array}{l}100 \\
400\end{array}$ & 400 & 400 & $R$ & $>800$ & 100 & $\mathrm{R}$ & - & $\mathrm{R}$ \\
\hline | & $R$ & $\begin{array}{l}50 \\
200\end{array}$ & 200 & $\mathrm{R}$ & Reg & 200 & 400 & - & 400 \\
\hline J & $\mathrm{R}$ & 50 & $>800$ & $\mathrm{R}$ & Reg & $>800$ & $>800$ & 100 & $\begin{array}{l}50 \\
400\end{array}$ \\
\hline
\end{tabular}

Comparison of spatial patterns ( $\chi^{2}$ contingency table): $M L$ vs. GL vs. WM, $c^{2}=5.28(6 D F, p>0.05)$

Vac-vacuolation, SN-surviving neurons, $G$ - glial cell nuclei, $P C$ - Purkinje cells, SC - surviving cells, GCI-glial cytoplasmic inclusions

Table IV. Frequency of correlations (Pearson's ' $r$ ') between histological features within and between layers of the cerebellum in multiple system atrophy. Figures indicate the number of cases in which a positive (+) or negative $(-)$ correlation was recorded

\begin{tabular}{|c|c|c|c|c|c|c|c|c|c|}
\hline \multicolumn{10}{|c|}{$\mathrm{X}$ variable } \\
\hline Y & MLV & MLN & MLG & PC & GLV & GLC & WMV & WMGCI & WMG \\
\hline MLV & - & $2(+)$ & - & $3(-)$ & $1(-)$ & - & - & - & $1(+)$ \\
\hline MLN & & - & $5(-)$ & $1(+)$ & $1(+)$ & - & $1(+)$ & $1(+)$ & $1(+)$ \\
\hline MLG & & & - & $1(-)$ & - & - & $1(+)$ & - & - \\
\hline PC & & & & - & $1(+)$ & - & - & - & $1(+)$ \\
\hline GLV & & & & & - & $5(-)$ & - & $1(+)$ & $2(+)$ \\
\hline GLC & & & & & & - & $1(+) 2(-)$ & - & - \\
\hline WMV & & & & & & & - & - & $1(+) 2(-)$ \\
\hline WMGCI & & & & & & & - & $1(+)$ & \\
\hline WMG & & & & & & & & & - \\
\hline
\end{tabular}

MLV - molecular layer vacuoles, MLN - molecular layer neurons, MLG - molecular layer glia, PC - Purkinje cells, GLV - granular layer vacuoles, GLC - granular layer cells, WMV - white matter vacuoles, WMGCI - white matter glial cytoplasmic inclusions, WMG - white matter glia

Spatial correlations among histological features within and between layers are summarised in Table IV. The most notable correlations were: (1) in the ML of 5 cases, a negative spatial correlation between glial cell nuclei and neurons, (2) in the GL of 5 cases, a negative spatial correlation between cells and vacuolation, and (3) in the PC layer of 3 cases, a negative spatial correlation between PC and vacuoles. Histological features in different layers of the cerebellar cortex were not spatially correlated. 
A PCA of the data resulted in the extraction of two PC accounting in total for $87 \%$ of the total variance $(P C 1=72 \%, P C 2=15 \%)$. A plot of the 10 cases in relation to $P C 1$ and $P C 2$ is shown in Fig. 7. MSA-C cases were located at the upper right of the plot and the MSA-P and cases of mixed pathology to the left of the plot. In addition: (1) PC1 was negatively correlated with the density of vacuoles in the ML $(r=-0.66, p<0.05)$ and (2) PC2 was negatively correlated with the density of cells in the $\mathrm{GL}(r=-0.81$, $p<0.05)$ and positively correlated with the density of vacuoles in the $\mathrm{GL}(r=0.82, p<0.001)$. In addition, cases to the right of the plot exhibited significant cerebellar ataxia but with minimum parkinsonism while those to the left of the plot exhibited a greater degree of parkinsonism, i.e. rigidity, akinesia, tremor, and less cerebellar ataxia.

\section{Discussion}

In the 10 MSA cases studied, a significant loss of $P C$ and vacuolation of the GL were the most consistent pathological changes compared with controls $[26,30,37]$. Some vacuolation was also evident in the $M L$ and white matter but at significantly lower levels than the GCL, and it did not differ quantitatively from controls. No $\alpha$-synuclein-immunoreactive inclusions were observed in control cases or in the grey matter of any MSA case, but such structures have been reported previously in the ML located into GFAP-immunoreactive radial processes of Bergmann glia [30,32]. $\alpha$-Synuclein-immunoreactive $\mathrm{GCl}$ were present in white matter, but not in all cases. GCl have been observed in other subcortical white matter tracts in MSA, including the external and internal capsules and central tegmental tract [9].

The vacuoles and surviving neurons were frequently clustered, and in some cases the clusters were regularly distributed relative to the edge of the folia, a pattern evident in both ML and GL. In addition, significant gaps were observed between PC perikarya, surviving PC often being regularly distributed, which suggests loss of clusters of PC. These results are consistent with a topographic pattern of the cerebellar pathology in MSA, which has also been observed in the cerebellum in the sporadic [7] and variant subtypes of Creutzfeldt-Jakob disease (CJD) [10]. A topographic loss of PC may also occur in Niemann-Pick type $C$ disease, in which there is a complex pattern of cell loss in the cerebellum, with surviving PC being aligned in strips [32]. Further loss

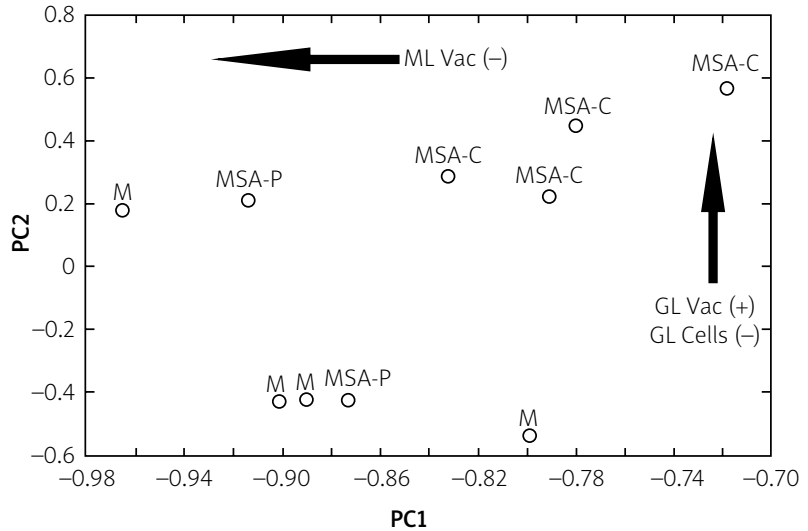

Fig. 7. Principal components analysis (PCA) of ten cases of multiple system atrophy (MSA) based on the densities of all histological features in the cerebellum. A plot of the cases in relation to PC1 and PC2 (MSA-C - cerebellar subtype, MSA-P - Parkinsonian subtype, $M$ - mixed pathology). Arrows PC1 was negatively correlated with the density of vacuoles in the molecular layer (ML Vac) and PC2 negatively correlated with the density of cells in the granule cell layer (GL Cells -) and positively correlated with the density of vacuoles in the GL (GL Vac + ).

of PC then occurs as the disease develops, resulting in large gaps between surviving cells similar to those observed in MSA.

There was a negative correlation between cells and vacuoles in the GL suggesting that vacuolation replaces lost neurons. Furthermore, there was a negative correlation between the densities of neuronal perikarya in the $M L$ and glial cell nuclei consistent with gliosis. A negative spatial correlation was also observed between individual PCs and clusters of vacuoles in the ML of three MSA-C cases, which could represent a more specific cerebellar pathology in MSA. These vacuoles may have developed in relation to the dendritic trees of the $P C$, which branch in a plane perpendicular to that of the section, the climbing fibres that ramify over individual PC, or the parallel fibres that ramify in the plane of the section and which are in contact with many adjacent PCs [10].

Although the number of cases of MSA is small, the PCA suggested some variations in quantitative pathology among cases. First, PC1 was negatively correlated with the density of vacuoles in the ML. Although there was no significantly increased vacuole density overall in the MSA cases, the vacuolation in the ML did vary among cases with more significant 
vacuolation in cases that exhibited more significant parkinsonism compared with those with cerebellar ataxia. Second, PC2 was negatively correlated with cell density and positively correlated with vacuole density in the GL. This result suggests that increased vacuolation and cell loss in the GL may be a more significant feature of the MSA-C subtype.

The cerebellum receives input from several sources (Fig. 8): (1) the spinal cord (posterior spino-cerebellar tract), reticular formation nuclei (reticulo-cerebellar tract), and pontine nuclei (ponto-cerebellar tract), which relay signals to the cerebellum, via the inferior and superior cerebellar peduncles, to the large diameter, rapidly conducting mossy fibres, the synaptic endings terminating in complex glomeruli; (2) climbing fibres that originate in the inferior olive (olivo-cerebellar tract) and which synapse directly on to the PC; and (3) fibres from the white matter which enter the GL and course parallel to the pia mater before synapsing with the PC [14]. $\alpha$-Synuclein-immunoreactive GCls have been observed in white and grey matter regions, which provide these inputs to the cerebellum in MSA, e.g. the ponto-cerebellar and reticulo-cerebellar tracts [13], and in the present study they were also observed in cerebellar white matter. This pathology has also been observed in motor tracts providing both the input and output pathways of the cerebellum, e.g., the cortico-pontine, cortical bulbar, cortico-spinal, and spino-reticular tracts. In addition, significant densities of inclusions have been observed in pre-cerebellar nuclei such as the inferior olivary nucleus [8], lateral reticular nucleus, interfascicular nucleus, and the nucleus of Roller in MSA [13].

Hence, $\alpha$-synuclein pathology spreading via cerebellar connections [35] could result in: (1) cell loss-

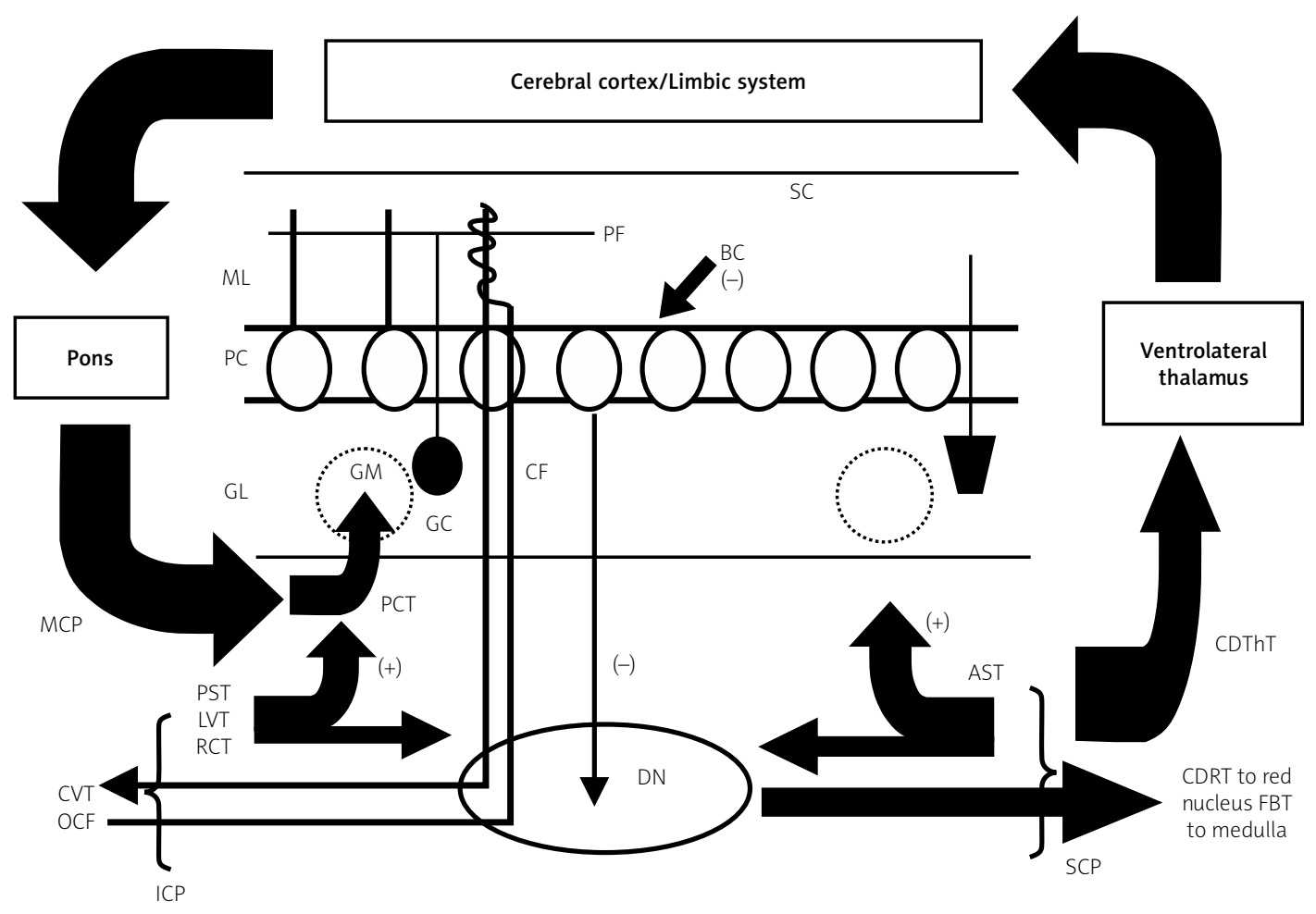

Fig. 8. Input and output pathways of the cerebellar cortex (ICP - inferior cerebellar peduncle, MCP - middle cerebellar peduncle, SCP - superior cerebellar peduncle, PF - parallel fibres, CF - climbing fibres, GM - glomerulus, BC - basket cell, GC - granule cell, SC - stellate cell, ML - molecular layer, PC - Purkinje cell layer, GL - granule cell layer, DN - dentate nucleus, ASCT - anterior spino-cerebellar tract, CDthT - cerebellodentato-thalamic tract, CDRT - cerebello-dentato-rubral tract, FBT - fastigio-bulbar tract, PCT - pontocerebellar tract, PST - posterior spino-cerebellar tract, LVT - lateral vestibular tract, RCT - reticulo-cerebellar tract, CVT - cerebello-vestibular tract, OCF - olivo-cerebellar tract (+ excitatory influence, - inhibitory influence). 
es and vacuolation in the GL, (2) loss of parallel and climbing fibres, (3) a reduction in the degree of facilitation of surviving PC, (4) a reduction in the degree of inhibitory control by PC of the dentate nucleus (DN), and (5) a reduction of fine tuning of the cerebral output via the cerebello-dentato-thalamic tract, which leaves the cerebellum as the superior cerebellar peduncle and connects the cerebellum to various regions such as the red nucleus (cerebello-dentato-rubral tract), medulla (fastigio-bulbar tract), and the cerebral cortex/limbic system, the latter- via the ventro-lateral thalamus. This pathology could potentially influence a variety of clinical symptoms reported in MSA, including dysfunction of motor activity, the fine timing of events, sensory analysis, feeding behaviour, the modulation of cognition, and in the regulation of emotions [22].

\section{Conclusions}

Cerebellar pathology in MSA may affect all layers of the cerebellar hemisphere, but cell losses and vacuolation in the GL and loss of PC were the most significant pathological changes in the cases studied. There was evidence of a topographic distribution of pathological change, which could reflect the spread of $\alpha$-synuclein pathology via anatomical connections. Cerebellar pathology may ultimately influence a variety of clinical symptoms in MSA, especially in the MSA-C subtype. Nevertheless, only 10 cases of this rare disorder were studied quantitatively, and these observations should be repeated on a larger series of well-characterised MSA cases.

\section{Acknowledgements}

Dr Diane Hanger is thanked for the generous donation of $\alpha$-synuclein antibody, and Heidi Barnes and Mavis Kibble for their excellent technical assistance.

\section{Disclosure}

Authors report no conflict of interest.

\section{References}

1. Armstrong RA. The usefulness of spatial pattern analysis in understanding the pathogenesis of neurodegenerative disorders, with particular reference to plaque formation in Alzheimer's disease. Neurodegeneration 1993; 2: 73-80.

2. Armstrong RA. Analysis of spatial patterns in histological sections of brain tissue. J Neurosci Meth 1997; 73: 141-147.
3. Armstrong RA. Quantifying the pathology of neurodegenerative disorders: quantitative measurements, sampling strategies and data analysis. Histopathology 2003; 42: 521-529.

4. Armstrong RA. Measuring the degree of spatial correlation between histological features in thin sections of brain tissue. Neuropathology 2003; 23: 245-253.

5. Armstrong RA. Methods of studying the planar distribution of objects in histological sections of brain tissue. J Microsc (Oxf) 2006; 221: 153-158.

6. Armstrong RA. Measuring the spatial arrangement patterns of pathological lesions in histological sections of brain tissue. Folia Neuropathol 2007; 44: 229-237.

7. Armstrong RA, Cairns NJ. Spatial patterns of the pathological changes in the cerebellar cortex in sporadic Creutzfeldt-Jakob disease (sCJD). Folia Neuropathol 2003; 41: 183-189.

8. Armstrong RA, Lantos PL, Cairns NJ. A quantitative study of the pathological changes in ten patients with multiple system atrophy (MSA). J Neural Transm 2004; 111: 485-495.

9. Armstrong RA, Cairns NJ, Lantos PL. A quantitative study of the pathological changes in white matter in multiple system atrophy. Neuropathology 2007; 27: 221-227.

10. Armstrong RA, Ironside JW, Lantos PL, Cairns NJ. A quantitative study of the pathological changes in the cerebellum in 15 cases of variant Creutzfeldt-Jakob disease (vCJD). Neuropathol Appl Neurobiol 2009; 35: 36-45.

11. Armstrong RA, Ellis W, Hamilton RL, Mackenzie IRA, Hedreen J, Gearing M, Montine T, Vonsattel J-P, Head E, Lieberman AP, Cairns NJ. Neuropathological heterogeneity in frontotemporal lobar degeneration with TDP-43 proteinopathy: a quantitative study of 94 cases using principal components analysis. J Neural Transm 2010; 117: 227-239.

12. Bower JH, Maraganore DM, McDonnell K, Rocca WA. Incidence of progressive supranuclear palsy and multiple system atrophy in Olmstead County, Minnesota, 1976-1990. Neurology 1997; 49: 1284-1288.

13. Braak H, Rub U, Del Tredici K. Involvement of pre-cerebellar nuclei in multiple system atrophy. Neuropathol Appl Neurobiol 2003; 29: 60-76.

14. Brodal A. Neurological anatomy. 3rd ed. Oxford University Press, New York, Oxford 1981.

15. Croisier E, Mres DE, Deprez K, Goldring K, dexter DT, Pearce RKB, Graeber MB, Roncaroli F. Comparative study of commercially available anti- $\alpha$-synuclein antibodies. Neuropath Appl Neurobiol 2006; 32: 351-356.

16. Dickson DW, Liu WL, Liu WK, Yen SH. Multiple system atrophy: a sporadic synucleinopathy. Brain Pathol 1995; 9: 721-732.

17. Dickson DW, Liu WK, Hardy J, Farrar M, Mehta N, Uitti R, Mark M, Zimmerman T, Golbe L, Sage J, Sima A, d'Amato C, Albin R, Gilman S, Yen SH. Widespread alterations of alpha-synuclein in multiple system atrophy. Am J Pathol 1999; 155: 1241-1251.

18. Ehot V, Brieger P, Broich K, Marneros A. Psychotic symptoms as initial manifestation of a multiple system atrophy. Fortshritte der Neurol Psych 1999; 67: 104-107.

19. Gilman S, Low PA, Quinn N, Albanese A, Ben-Schlomo Y, Fowler CJ, Kaufmann H, Klockgether T, Lang AE, Lantos PL, Livan I, Mathias CJ, Oliver E, Roberstson D, Schatz I, Wenning GK. Con- 
sensus statement on the diagnosis of multiple system atrophy. J Auto Nerv Syst 1998; 74: 189-192.

20. Gilman S, Low PA, Quinn N, Albanese A, Ben-Schlomo Y, Fowler CJ, Kaufman H, Klockgether T, Lang AE, Lantos PL, Litvan I, Mathias CJ, Oliver E, Robertson D, Schatz I, Wenning GK. Consensus statement on the diagnosis of multiple system atrophy. J Neurol Sci 1999; 163: 94-98.

21. Gilman S, Wenning GJK, Low PA, Brooks DJ, Mattias CJ, Trojanowski JQ, Wood NW, Colosima C, Durr A, Fowler CJ, Kaufmann H, Klockgether T, Lees A, Poese W, Quinn N, Revesz T, Robertson D, Sandroni T, Seppi K, Vidailhet M. Second consensus statement on the diagnosis of multiple system atrophy. Neurology 2008; 71 : 670-676.

22. Ioannides AA, Fenwick PBC. Imaging cerebellum activity in real time with magnetoencephalographic data. In: Creating Coordination in the Cerebellum. Prog in Brain Res 2005; 148: 139-150.

23. Konogaya M, Sakai M, Matsuoka Y. Konogaya Y, Hashzume Y. Multiple system atrophy with remarkable frontal lobe atrophy. Acta Neuropathol 1999; 97: 423-428.

24. Kovacs GG, Milenkovic IJ, Preusser M, Budka H. Nigral burden of alpha-synuclein correlates with striatal dopamine deficit. Move Disord 2008; 23: 1608-1612.

25. Lantos PL. Cellular pathology of multiple system atrophy: a review. J Neurol Neurosurg Psychiatr 1994; 57: 129-133.

26. Mori F, Piao YS, Hayashi S, Fujiwara H, Hasegawa M, Yoshimoto M, Iwatsubo T, Takahashi H, Wakabayashi K. Alpha-synuclein accumulates in Purkinje cells in Lewy body disease but not in multiple system atrophy. J Neuropathol Exp Neurol 2003; 62: 812-819.

27. Oertel WH, Bandmann O. Multiple system atrophy. J Neural Transm (Suppl) 1999; 56: 155-164.

28. Papp MI, Kahn JE, Lantos PL. Glial cytoplasmic inclusions in the CNS of patients with multiple system atrophy (striatonigra degeneration, olivopontocerebellar atrophy and Shy-Drager syndrome). J Neurol Sci 1989; 94: 79-100.

29. Papp MI, Lantos PL. The distribution of oligodendroglial inclusions in multiple system atrophy and its relevance to clinical symptomology. Brain 1994; 117: 235-243.

30. Park SH, Becker-Catania S, Gatti RA, Crandall BF, Emelin JK, Vinters HV. Congenital olivopontocerebellar atrophy: report of two siblings with paleo and neocerebellar atrophy. Acta Neuropathol 1998; 96: 315-321.

31. Piao YS, Mori F, Hayashi S, Tanji K, Yoshimoto M, Kakita A, Wakabayashi K, Takahashi H. Alpha-synuclein pathology affecting Bergmann glia of the cerebellum in patients with alpha-synucleinopathies. Acta Neuropathol 2003; 105: 403-409.

32. Sarna JR, Larouche M, Marzban H, Sillitoe RV, Rancourt DE, Hawkes R. Patterned Purkinje cell degeneration in mouse models of Niemann-Pick type C disease. J Comp Neurol 2003; 456 : 279-291.

33. Sebeo J, Hof PR, Perl DP. Occurrence of alpha-synuclein pathology in the cerebellum of Guamanian patients with parkinsonism dementia complex. Acta Neuropathol 2004; 107: 497-503.

34. Spillantini MG, Crowther RA, Jakes R, Cairns NJ, Lantos PL, Goedert M. Filamentous $\alpha$-synuclein inclusions link multiple system atrophy with Parkinson's disease and dementia with Lewy bodies. Neurosci Lett 1998; 251: 205-208.
35. Steiner JA, Angot E, Brunden P. A deadly spread: cellular mechanisms of $\alpha$-synuclein transfer. Cell Death and Differ 2011; 18: 1425-1433.

36. Wakabayashi K, Ikeuchi T, Ishikawa A, Takahashi H. Multiple system atrophy with severe involvement of the motor cortical areas and cerebral white matter. J Neurol Sci 1998; 156: 114-117.

37. Wenning GK, Tison F, Ben-Shlomo Y, Daniel SE, Quinn NP. Multiple system atrophy: a review of 203 pathologically proven cases. Move Disord 1997; 12: 133-147. 